\title{
Large Area, High-resolution Multilayered Imaging Approach Using Block-face SEM: Identification of Neuro-degeneration in Mouse Model of 22q11 Deletions Syndrome
}

\author{
C. A. Brantner ${ }^{1}$, P. Mistry ${ }^{1}$, L. Matsiyevskiy ${ }^{1}$, C. Bryan ${ }^{2}$, D. Meechan ${ }^{2}$, T. M. Maynard ${ }^{2}$ and A. \\ Popratiloff ${ }^{3}$. \\ 1. Nanofabrication and Imaging Center, George Washington University, Washington, DC USA. \\ 2. School of Medicine and Health Sciences, George Washington University, Department of \\ Pharmacology, Washington DC USA. \\ 3. Institute for Neuroscience, Program for Pediatric Dysphagia, George Washington University, \\ Washington DC USA.
}

Electron microscopy has been a prime and valuable tool for neuroscience discoveries, but a tendency has developed to substitute EM approaches with more "user friendly" but resolution limiting optical imaging approaches, such as confocal microscopy. The strengths of the latter are that it can provide images of the cellular detail and overall tissue context simultaneously. The neuroscience community is beginning to broadly appreciate the need for high-resolution image data, and at the same time electron microscopy technology has became more supportive of integrating imaging modalities such as integration of low and high-resolution data. Although the traditional electron microscopy can provide very high-magnification and high-resolution imaging data, sufficient to resolve the smallest cellular organelles, frequently it is very difficult to correlate subcellular changes with the overall tissue context. This difficulty is amplified when a rare pathological event needs to be identified. In such cases, relying on finding that rare event on a TEM grid is like a fishing expedition. Ideally, a zoomable, Google map-type of electron microscopy image data could serve such a need (i.e. similar to virtual light microscopy slides achieved with slide scanner or optical microscopes equipped with scanning stage). Here we present an integrated workflow that allows capturing and analyzing large areas of the brainstem while maintaining high cellular resolution and specificity of the imaged area.

Our initial confocal data, using a mouse model of the human disease known as DiGeorge Syndrome (22q11 deletion, LgDel), indicated a possibility of neuronal degeneration in the hypoglossal nucleus. This group of motor neurons that innervate the tongue muscles are likely compromised in the LgDel, since human patients carrying the phenotype, frequently have swallowing and feeding challenges. We used ultrathin sections $(100 \mathrm{~nm})$ from the entire brainstem mounted on silicon wafer to generate highresolution images from the hypoglossal nucleus of wild type (WT) and LgDel mice. Initially, $400 \mu \mathrm{m}$ brainstem sections were fixed in osmium tetroxide, infiltrated with aqueous uranyl acetate, dehydrated and flat embedded in resin. Sections $(4 \times 3 \mathrm{~mm})$ were cut with extra-large jumbo histo diamond knife and placed on silicon wafer for imaging with FEI Helios FIBSEM equipped with circular backscattering (CBS) detector. The entire cross-section of the brainstem was first imaged at a magnification of 600X, which served as a low-resolution map to select areas of interest for high-resolution imaging. An area of the hypoglossal nucleus was then imaged at 1000X for initial cellular resolution, from which an area of $418 \times 241 \mu \mathrm{m}$ was selected for imaging at high-resolution. The high-resolution imaging was done using FEI MAPS software. Tile-scan images are taken at the following parameters: magnification $-80,000 \mathrm{X}$; accelerating voltage - $2 \mathrm{kV}$; landing current - 200 pAmps; Pixel dwell time - 3 usec. Individual images were $3072 \times 2048$ pixels, with each pixel measuring $2.095 \mathrm{~nm}$, providing horizontal field of view of 6.4 $\mu \mathrm{m}$. Adjacent images were overlapped $20 \%$ for proper stitching. The same size area was captured from 
WT and LgDel hypoglossal nuclei. Stitched images were exported from MAPs software as *.RAW format and opened in Arivis for manual segmentation and annotation.

Electron microscopy image data clearly defined neuronal degeneration in the hypoglossal nucleus of LgDel mice. Analyzing EM data from an area representing 2/3 of the one-sided hypoglossal nucleus, one neuron was found in the initial stages of degeneration, while three others in more advanced stages were seen. The most striking finding, however, was that all hypoglossal motor neuorns displayed mild neurodegenerative changes and consistent from cell to cell phenotype of endoplasmic reticulum and Golgi apparatus stress. Synapses and mitochondria both showed a decrease in number and an increase in abnormality. Our approach enabled us to identify a novel mechanism underlying feeding and swallowing deficits in the mouse model of the DiGeorge Syndrome.

\section{References:}

[1] A Lamantia et al, Dev. Biol. 409(2) (2016), p. 329.
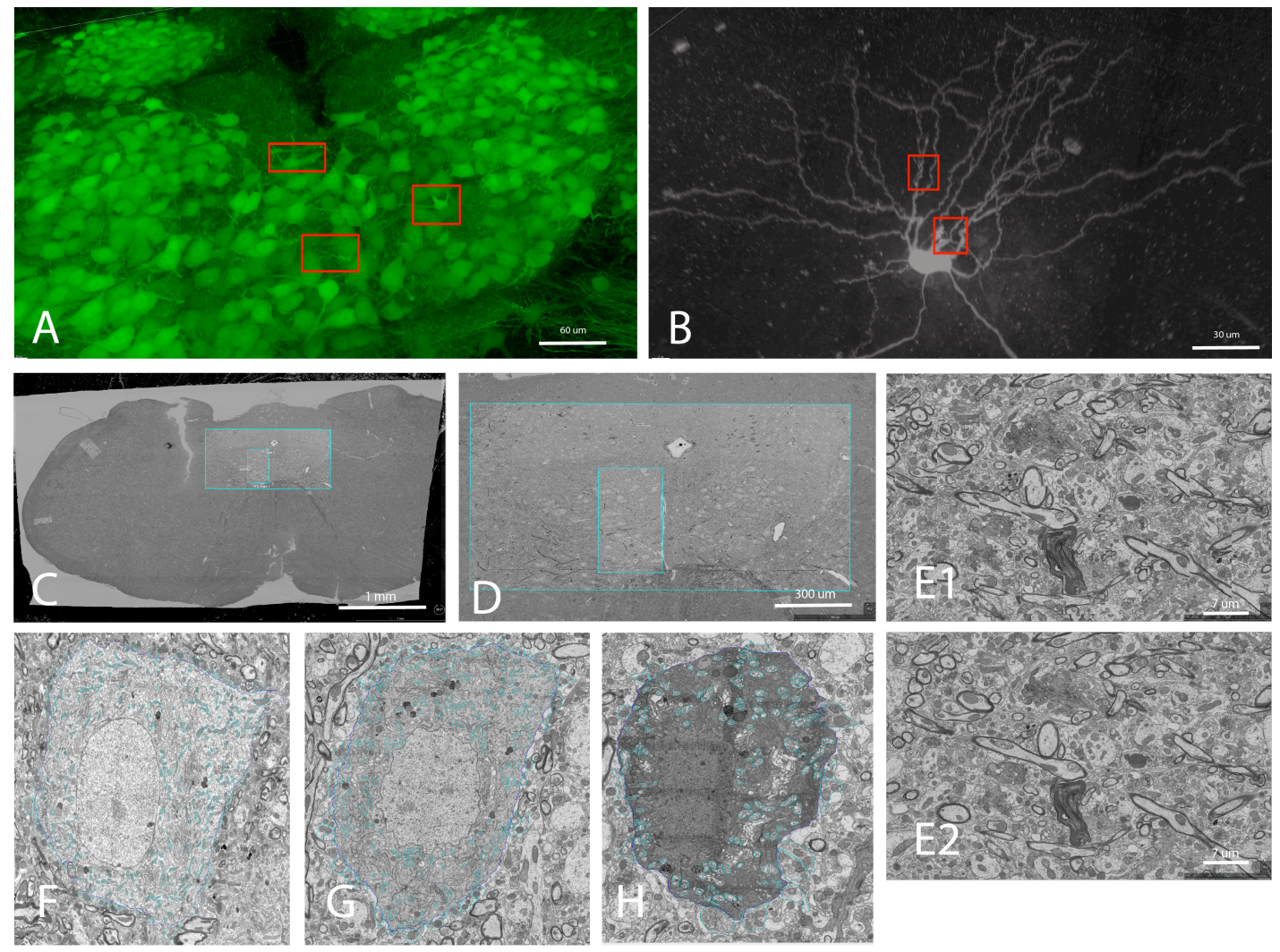

Figure 1. A. $3 \mathrm{D}$ confocal images from $\mathrm{LgDel} / \mathrm{ChAT}$ reporter mouse showing a few abnormal motor neurons (red boxes). B. Biocytin injected neuron with several corkscrew dendrites, characteristic of neurodegeneration. C. SEM tile-scan at low-resolution covering the mouse brainstem. Boxes indicate areas selected for high-resolution imaging. D. Area of XII nucleus, the larger box shows the area taken at 1000X, while the inner, smaller box shows the area taken at 80,000X. E1 and E2. Show scanning errors (E1) and their correction after stitching (E2). F-H. XII neurons at different stages of degeneration (early $-\mathbf{F}$, moderate $-\mathbf{G}$, severe $-\mathbf{H}$ ), also in color are shown mitochondria and synapses segmented using Arivis software. 